\title{
Air-Core Sensors Operation Modes for Partial Discharge Detection and On-line Diagnostics in Medium Voltage Networks
}

\author{
Lauri Kütt (Researcher, Aalto University School of Electrical Engineering - AUSEE), \\ Muhammad Shafiq (Researcher, AUSEE), Matti Lehtonen (Professor, AUSEE), \\ Heigo Mõlder (Researcher, Tallinn University of Technology - TUT), Jaan Järvik (Professor, TUT)
}

\begin{abstract}
In this paper, sensors for the application of high voltage networks insulation diagnostics are focused on. For detection and localization of the components with insulation deterioration, observing the partial discharge transients is one of the main methods. However, as the partial discharge traces are transients of extremely short duration, monitoring of such transients is also a challenging task. This paper presents the inductive sensors that would be suitable for the on-line partial discharge monitoring and the selection of operating mode and characteristics are discussed. The issues of resonance and signal processing are analyzed for the targets of reaching highest accuracy of the measurements. Operation criteria and performance limits such as sensitivity and upper bandwidth are particularly discussed. It is presented that in order to reach highest accuracy and sensitivity the sensor should be used in damped mode. Applying the accurate air-core inductive sensors would allow the condition monitoring systems to be cheaper and thus mounted for permanent and continuous condition monitoring in various locations in the power distribution networks.
\end{abstract}

Keywords - Power distribution faults, current measurement, pulse measurements, partial discharge measurement.

\section{INTRODUCTION}

A distribution network, consisting of high number of components, can suffer a fault and power outage in case of even single component like insulator fails. Complete failure of a component will very likely lead to switch-off of the power network and a long supply interruption for the customers, leading to rise of customer dissatisfaction [1]. If however the components likely to fail are detected and located in advance, the components could be repaired or replaced with least time and previously notified shorter interruption. Today, the on-line methods allowing the condition monitoring to be performed at the same time when the network is in its normal operation state are more feasible for diagnostics. Such methods would allow most effective diagnostics as the line is observed continuously in operational state and identify the line condition under the operational stress (high voltage, large currents).

For the high-voltage networks, loss of insulation is one of the most frequent causes for failures. In order to detect insulation problems, observing the partial discharge activity [2] has been presented as one of the most suitable ones. Partial discharge events are related only to a momentary transfer of charge and the magnitude of charge transferred during the partial discharge event depends on the voltages used in the power network. If there is some damage to the insulation, intense and growing PD activity will be observed [3]. The medium-voltage distribution networks which supply a relatively high number of customers and use voltages higher than $3 \mathrm{kV}$, such partial discharge events provide significant magnitude of transients and these can be detected relatively easily from a distance by using suitable sensors. Still, the displacement of charge during the partial discharge lasts only for a miniscule time and its electrical trace is a current pulse with same duration.

Classical substation designs include voltage and current transformers with ferromagnetic cores and are mostly intended for measurement of quantities at mains frequencies (50 or $60 \mathrm{~Hz}$ ). Such transformers are known for their limited capabilities while measuring even the mains harmonics [4]. The short-duration partial discharge traces however include different high-frequency components, reaching into tens and even hundreds $\mathrm{MHz}$ [5]. This is where the wide-bandwidth air-core inductive sensors provide outstanding performance. Implementing the inductive sensors for partial discharge measurements has been proposed also before [6][7] with sensors indicated bandwidths of up to several hundreds of $\mathrm{MHz}$ [8], although the role of resonance and the measurement system characteristics has generally been neglected. The possible different operating modes of an inductive sensor have also been presented in [9] while criteria for the pulsed phenomena measurements have not been clearly identified in the context.

In this paper the sensor bandwidth, operating modes and expected performance are discussed for the air-core inductive sensor implementation for partial discharge on-line measurements. Nevertheless, similar criteria apply for other pulsed current measurements. For establishing the criteria for the measurement, an introduction to the basic terms of partial discharge and diagnostics based on observing the partial discharge intensity is presented. The sensor operation is discussed along with the most common operating modes, which also would be the most probable ones for the inductive sensor implementation. Different operating modes are simulated and the comparison of the expected measurement outcome presented. For the sensor operation verification, comparison of the measurements carried out in laboratory will be provided. 


\section{CONDITION MONITORING USING PARTIAL DischargeS}

The role of a proper high-voltage insulation material is to keep the charges from passing through the material. However, if it has lost some of its insulation capability the charges could be able to penetrate through with the force provided by the high electric field strength. When the insulation material deterioration is minor but with some effect, small localized charge transfer events called partial discharges (PD) occur due to defects (cavities, voids, cracks or inclusions) of solid or liquid insulation material which is under high voltage stress [10]. A PD phenomenon is only momentary (having nano to micro second duration) and does not result in high currents and arcing. The intensity of a PD is dependent on the electric field gradients in the material. Presented in the histogram in Fig. 1, the partial discharge magnitudes have been plotted in respect to the high voltage mains cycle. The most intensive PD is seen when the voltage between the conductors is high (near peaks) and the voltage absolute value is increasing.

As a result of such small charge transfer occurrence, voltage between the conductors drops to some extent, due to the decrease of charge difference between the conductors. Associated with the movement of charge, current is also present and fast transient voltage and current pulse is created with the duration of some nano to micro-seconds. Short pulses can have excellent propagation characteristics since they essentially are electromagnetic waves traveling along the power line at speed of light.

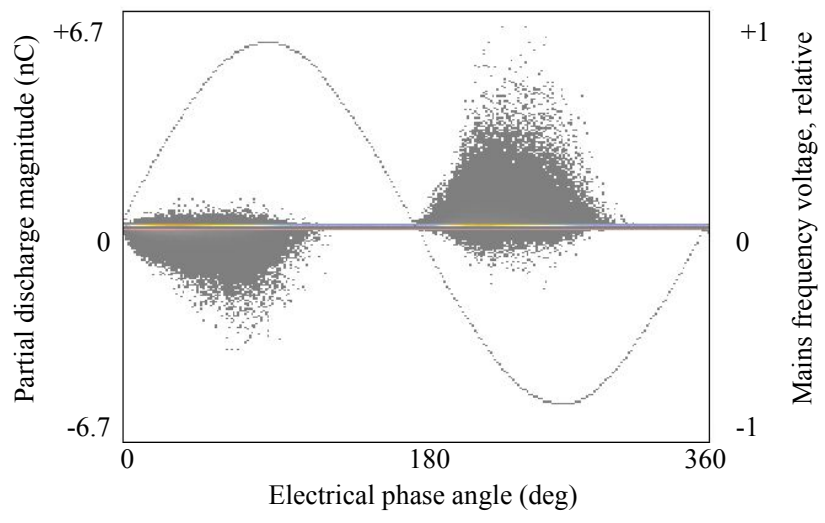

Fig. 1. Partial discharge activity measured during a cycle of supply voltage.

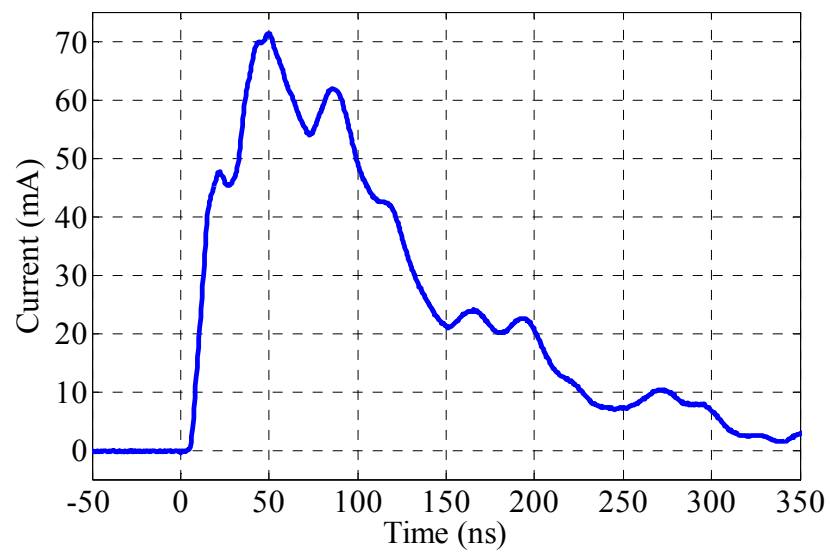

Fig. 2. Example of a partial discharge pulse.
An example of a PD-pulse is presented below in Fig. 2, originating from a laboratory test set-up and PD-calibrator. Practical measurements have confirmed that it is rather identical to the actual average PD-waveform. This pulse has been measured using an accurate calibrated high-frequency current transformer having $80 \mathrm{MHz}$ upper bandwidth limit. This PD-pulse will be used as a reference pulse in the following discussion. The duration of a PD pulse is dependent on the partial discharge magnitude, evaluated in units of charge. The sample partial discharge pulse in Fig. 2 has magnitude of $23 \mathrm{nC}$. It can be observed that this pulse has a rise time of less than $50 \mathrm{~ns}$ and total duration of about $300 \mathrm{~ns}$. The peak intensity of the PD transient current in range of tens $\mathrm{mA}$ reveals the requirements for the sensor sensitivity.

While sine waveforms are having defined frequency, each pulsed signal consists of a high number of frequency components. Especially the short-duration pulsed transients could have bandwidth reaching into tens or hundreds $\mathrm{MHz}$. This sets also the requirements for the sensor operation, used to measure the pulsed phenomena. Accuracy is guaranteed only provided all of these frequency components are measured with same sensitivity and zero phase shift. Thus, for measurements of the short-duration pulsed transients, a sensor is needed that would allow measurements in frequency ranges up to at least tens of $\mathrm{MHz}$ and having stable magnitude and phase transfer.

Measurement of these extremely fast pulses in substations can provide the information where the insulation degradation is happening, how far it has progressed and how fast it is progressing. After the signal has been acquired from the physical measurements, it can be passed on to the diagnostics system consisting of more complex data storage and processing units. The overall layout of such on-line diagnostic systems has been provided in Fig. 3. More detailed pulse characteristics can be derived for the evaluation of, for example, defect type, location, evolution and many others. For this different pulses from different sources should be identified and compared over a longer period of time. For more accurate diagnostics more accurate measurements are required.

As a result, the information of the possible insulation problems is passed to the network operator for issuing repairing procedures. If a potential fault is detected prior to complete breakdown it can help avoiding significant economic loss and customer dissatisfaction [11].

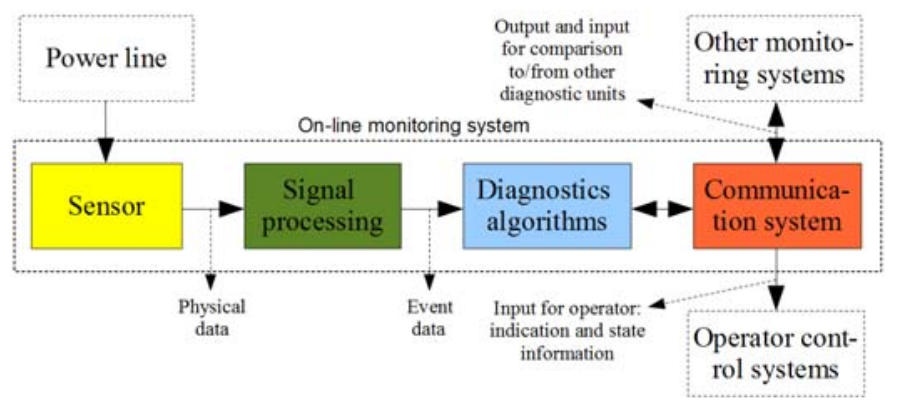

Fig. 3. Comparative waveforms of measurements of a real PD pulse. 


\section{IndUCtIVE SENSOR PRINCIPLES}

Every wire that has a current passing through it provides some magnetic field around it. The relation of magnetic field intensity and magnetic flux density around current-carrying wire is well-known and can be [12] found in many textbooks

$$
B=\mu \cdot H=\frac{\mu \cdot I}{2 \pi \cdot r},
$$

where $I$ - current passing through the wire, $r$-distance from the wire to the observation point and $\mu$ - relative magnetic permeability.

Passing through some area $A$, the area holds magnetic flux

$$
\Phi=\int_{A} B d a,
$$

where $d a-$ smallest section of area $A$. This refers that magnetic flux through the loop that is in the vicinity of a wire with current depends on the loop geometrical parameters and position.

Inductive current sensor relies on the Faradays law, stating that variable magnetic flux, passing through a conductive loop, provides electromotive force in the ends of the loop as

$$
e=-\frac{d \Phi}{d t}
$$

where $e$ - electromotive force (EMF); $\Phi$ - magnetic flux through loop; $t$-time. This means that the electromotive force depends on the rate of change of the flux linkage acquired by the coil loop area. It provides a simple relation - the faster the change, the more there is output EMF. For making a sensor system, a loop of wire, placed in the area with changing magnetic field effect and a sensitive voltmeter, connected to the ends of the loop, as in Fig. 4 would be used.

In defining the magnetic field effect towards the sensor, a factor of mutual inductance would be used. The mutual inductance $M$ is dependent on the geometry of the sensor and also the number of loops $N$ of the sensor connected in series

$$
M=N \int_{A} \frac{\mu}{2 \pi r} d a .
$$

The output of the sensor can be expressed as

$$
e_{\text {out }}=-M \frac{d i_{\text {line }}}{d t} .
$$

The sensitivity of the sensor is directly related to the loop geometrical shape. For some most common loop shapes, the mutual inductance calculations are presented in [13].

An example of a practical inductive sensor, also referred to later in this work is the Rogowski coil (see Fig. 5). It has a winding constructed on a non-magnetic core, with turns wound in toroidal manner. When Rogowski coil is mounted in a way that the current-carrying wire would be in the center of the sensor, all the turns are located perpendicular towards the current wire being measured.

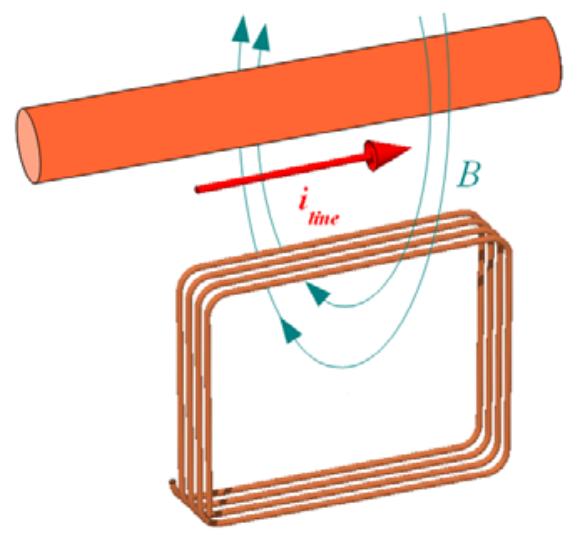

Fig. 4. Inductive current sensor operating principle.

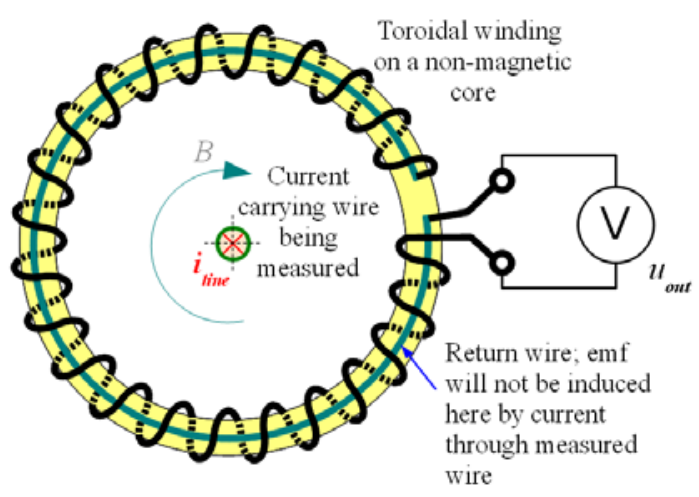

Fig. 5. Rogowski type inductive current sensor.

The return wire in the center of winding (see Fig. 5) would be beneficial for canceling out the effects of external fields not originating from the current in the wire. Using the return wire allows also openable Rogowski coils, making the assembly around existing primary current carrying wire extremely simple.

There are several advantages that the inductive sensors have for the high voltage applications. First, the inductive sensors offer possibility for totally uncoupled measurements. For high voltage systems this has high importance as any device directly coupled to the HV system needs strong and expensive insulation. The air-core inductive sensors also have very fast response and no saturation, providing a wide linear operating bandwidth.

The air-core inductive sensor can be designed to have high sensitivity either in the low frequency range or high frequency range. The application of PD transients measurement requires the sensor to be capable of performing the measurements in the high frequency range. The sensor designed for this purpose will have negligible sensitivity for the mains frequency current passing through the wire. This means that the air-core sensor does not eliminate the need for the ferromagnetic transformers in the substations for the measurements of mains frequency quantities. Instead, the air-core sensor would be an addition to the classical substation design, independent of the other control functions. 


\section{High-FrequenCy SENSOR CHARACTERISTICS AND CONNECTION TO THE MEASUREMENT SYSTEM}

The Faradays law (4) states that the faster is the rate of change of the magnetic flux, the higher is the output EMF magnitude. This means that the output of the sensor is higher as the frequency of the measured current increases. It has to be kept in mind that the differential relation (5) is valid only in the case of the sensor ideal standalone operation. In case of real world high frequency measurement application, external components will define a significant portion of the sensor's operation characteristics. However, the sensor itself has also principal electrical characteristics that have to be accounted.

The inductive sensor coil possesses self-inductance and self-capacitance. These are the essential quantities defining the self-resonance frequency (see (7)) and also the bandwidth of the sensor (discussed later in this paper). In addition, the copper wire has some active resistance, though this is very small for a low-turn, low-inductance sensor. Such components are represented as $L_{S}, C_{S}$ and $R_{S}$ respectively in the equivalent circuit described in Fig. 6, appropriate for observing the highfrequency operation of a sensor coil. With operating frequencies reaching close to and beyond $100 \mathrm{MHz}$, the equivalent circuit will be even more complex [14].

The measurement system layout equivalent circuit for the power line diagnostic measurements using the inductive sensors has been presented in Fig. 6. Here, current monitored in the power line provides an EMF source associated with the mutual inductance $M_{S}$ between the sensor coil and the wire being measured. Added to the circuit directly across sensor coil terminals is a terminating resistor $R_{T}$, which provides active load for damping the resonant oscillations of the circuit. While its role has not been emphasized [6][7], it is a major component that can define the sensor operating mode.

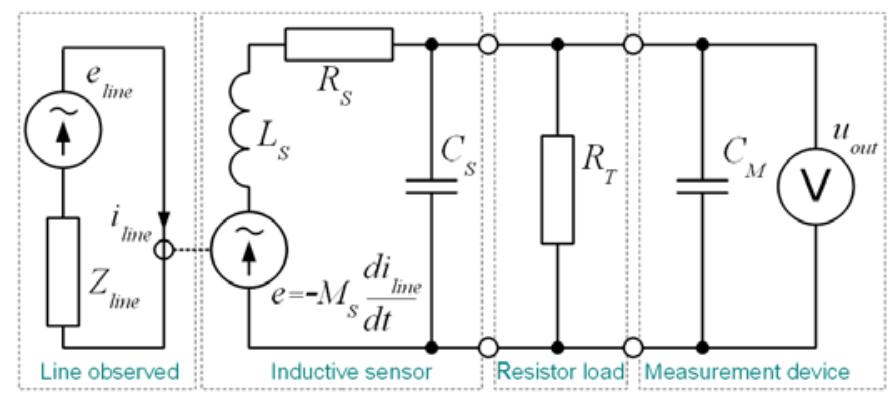

Fig. 6. Equivalent circuit of the inductive sensor measurement system.

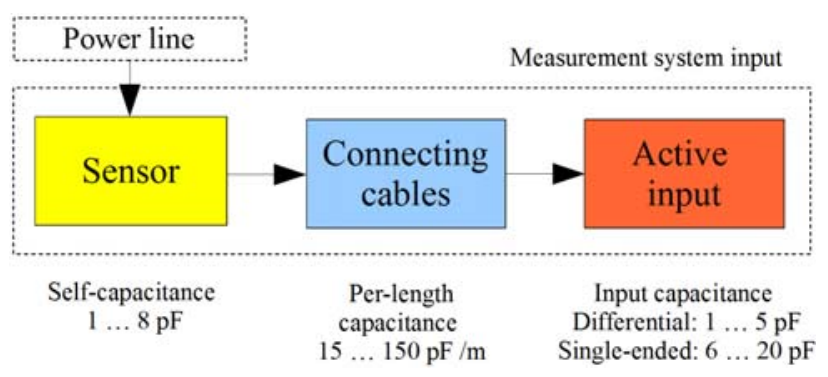

The inductive sensor system output is an active measurement device, represented as a voltmeter in Fig. 6. This represents an active component, for example an active amplifier [8] or analog-to-digital converter [15] that provides very small loading for the circuit and decouples the sensor circuit from the rest of the data processing system. It can be assumed that the input resistance of an active unit reaches very high values (mega-ohms) and therefore has not been represented as a separate resistive component. However, this connection provides also input capacitance and active resistance of the measurement device. Even the cables used to connect the sensor to the measurement system input have some capacitance. Capacitances of the measurement system as well as the connecting cables are represented as total equivalent capacitance $C_{M}$.

All the capacitances between the sensor output terminals are in parallel and will add to the overall system and sensor capacitance value (see Fig. 7). The higher capacitance value results in lower resonant frequency and lower operating bandwidth higher frequency. For the high frequency operation, if possible, it should be avoided that the external circuit had several times higher capacitance than the sensor coil itself. This means using as short connections as possible and lowcapacitance probes.

For more detailed sensor system operation analysis in wide frequency range it is more appropriate to use a transfer function in $s$-domain. The transfer function for the circuit presented in Fig. 6 can here be expressed as [14]

$$
U_{\text {out }}(s)=\frac{s \cdot M_{S} \cdot I_{\text {line }}(s)}{L_{S} \cdot C_{C}\left[s^{2}+\left(\frac{1}{R_{T} \cdot C_{C}}+\frac{R_{S}}{L_{S}}\right) s+\left(\frac{1+\frac{R_{S}}{R_{T}}}{L_{S} \cdot C_{C}}\right)\right]},
$$

where $C_{C}$ is a sum of capacitances $C$ and $C_{M}$ connected in parallel.

Common to a RLC circuit, the transfer function is a second order function which can have oscillations in its transient response. There will be 2 poles for the transfer function (6), defining the resonance conditions. In addition, there is a zero which is due to differentiating nature of the sensor. Values of the poles provide information about the sensor set-up behavior and can be found by solving the function of variable $s$ found in the denominator.

\section{V.Operating MODES OF THE INDUCTIVE SENSOR}

For characterizing the inductive sensor high-frequency operation, three main operation modes could be identified. These are resonant operation, critically damped operation and overdamped operation modes. The selection of the mode of operation can be tuned using external components, especially the terminating resistor $R_{T}$. Each operation mode and the sensor performance in such mode is described below.

Fig 7. Probable capacitance values in the inductive sensor application. 


\section{A. Resonant Operation}

An example of the resonant mode of operation is observed when the sensor coil is connected to an oscilloscope probe without connecting $R_{T}$. The probes used with oscilloscopes usually have very high input resistance, commonly in the range of $1 \ldots 10 \mathrm{M} \Omega$. The current through such resistance is very small and the energy is not dissipated from the LC circuit. In this case the poles of the transfer function form a conjugate complex pair, which results in heavy oscillations in the output of the system. Such oscillations occur at the frequency defined by the sensor coil self-resonance and selfcapacitance as

$$
f_{\text {res }}=\frac{1}{2 \pi \sqrt{L_{S} C_{C}}} .
$$

The resonant mode operation would provide the highest sensitivity for the sensor as there is only slight load connected to the sensor. The transfer rate is significantly higher for the frequencies close to the resonant frequency value. This will emphasize frequency components close to $f_{\text {res }}$ in the measurement result.

Beyond the $f_{\text {res }}$, however, the transfer rate will drop sharply in linear relation to the frequency (see Fig. 8).

\section{B. Critically Damped Operation}

Adding a lossy resistor load $R_{T}$ will introduce the component to dissipate the energy from the circuit. The resonant oscillations are weaker or even no longer present as the energy stored in $L_{S}$ and $C_{S}$ is dissipated from the resistor. This will lead to the inductive sensor system output that is close to ideal differential value of the current being measured (see (5)). At the same time the sensitivity of the sensor would remain basically at the same level as for the resonant operation mode. It can also be observed that the magnitude transfer rate is still the highest at the resonant frequency value.

For reaching the critically damped mode operation, when the oscillations are no longer present in the sensor output, the selection of the $R_{T}$ is important. Damping rate $\xi$, dependent on the $R_{T}$ value can be found as

$$
\xi \approx \frac{1}{2 \cdot R_{T}} \sqrt{\frac{L_{S}}{C_{C}}} .
$$

Critical damping is reached when the damping coefficient is exactly 1 . For this, the damping resistor should have a value of

$$
R_{T} \approx \frac{1}{2} \sqrt{\frac{L_{S}}{C_{C}}} .
$$

Critical damping mode of operation is useful for the application as it would provide the output of the sensor with the differential value of the current measured (this is still valid until reaching frequency of $f_{\text {res }}$ ). Later in the signal processing stages the integration operation would be performed to obtain the original measured current waveform.

\section{Overdamped Operation}

The overdamped operation mode is reached when the sensor load is further increased i.e resistance of $R_{T}$ is further decreased. While for the resonant and critically damped modes the 2 poles of the transfer function (6) were at the same frequency value, the overdamped mode will present the poles with different values [16]. The location of poles will be further away from each other as the load of the sensor is increased. This however will offer a benefit also for the pulsed current measurement. Namely, in the frequency range from the lower frequency pole to the higher frequency pole, the sensor output is not differential of the current measured, but instead the direct value of the measured current. Therefore the sensor will provide the output proportional to the actual value of the measured current without the external integrator. For this reason, the operation in this region would also be called "selfintegrating" operation [9].

\section{Comparison of the Operating Modes}

Fig. 8 presents the magnitude transfer plots for the different operating modes, using the same $L_{S}$ and $C_{C}$ values while value of $R_{T}$ is varied. The slope $+20 \mathrm{~dB}$ (10 times) per frequency 10 time (decade) increase presents the differentiating nature of the sensor system output. For the easier visualization, the plot has been normalized so that the damped mode provides highest transfer rate of $1(0 \mathrm{~dB})$. Increase of transfer rate for a particular frequency represents the resonant operating point and it can be seen that the transfer rate starts to drop immediately after the resonant frequency. Beyond this frequency, the higher frequency components will not be equally present, even after the integration, in the output signal of the system. The overdamped mode can be observed to provide lower transfer rate than the other modes.

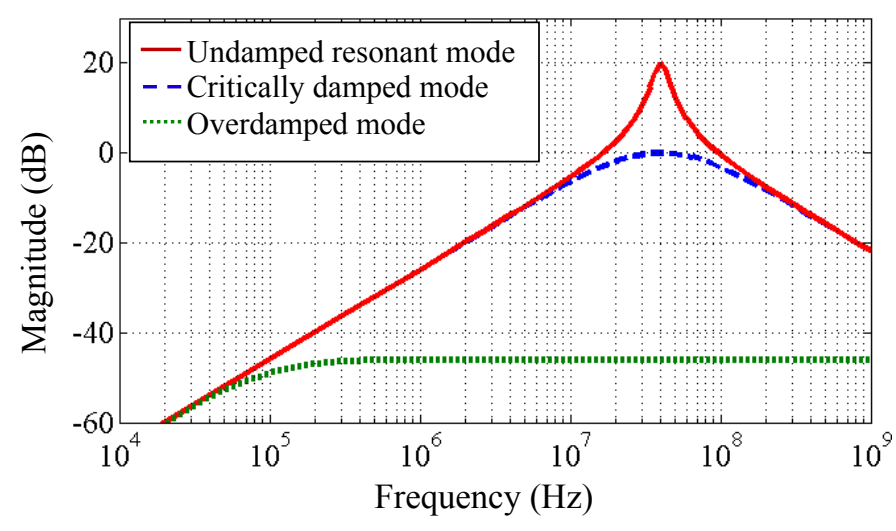

Fig. 8. Magnitude transfer plots for resonant, damped and overdamped sensor operating modes.

\section{Sensor Output Signal Processing}

For the resonant and also the damped modes of operation, the sensor's output voltage value would be proportional to the differential of the instantaneous measured current value, as observed from (5), until $f_{\text {res }}$. In order to restore the original measured current waveform, the output would have to be integrated to cancel out the differentiating effect [15] 


$$
i_{\text {res }, \text { int }}=\int u_{\text {out }} d t=-M_{S} \int \frac{d i_{\text {line }}}{d t} d t=-M_{S} \cdot i_{\text {line }} .
$$

This integration operation could be performed using analog active circuits, connected to the sensor as active input, or digital signal processing units applied to process the results from the analog-to-digital converter.

Prior to performing the integration it is rather difficult to define the absolute sensitivity of the sensor, as the output voltage of the sensor would be directly dependent on the frequency of the current components measured. However, after the integration, the output of the sensor is rather independent of the frequency of the particular measured frequency component, until the resonant frequency.
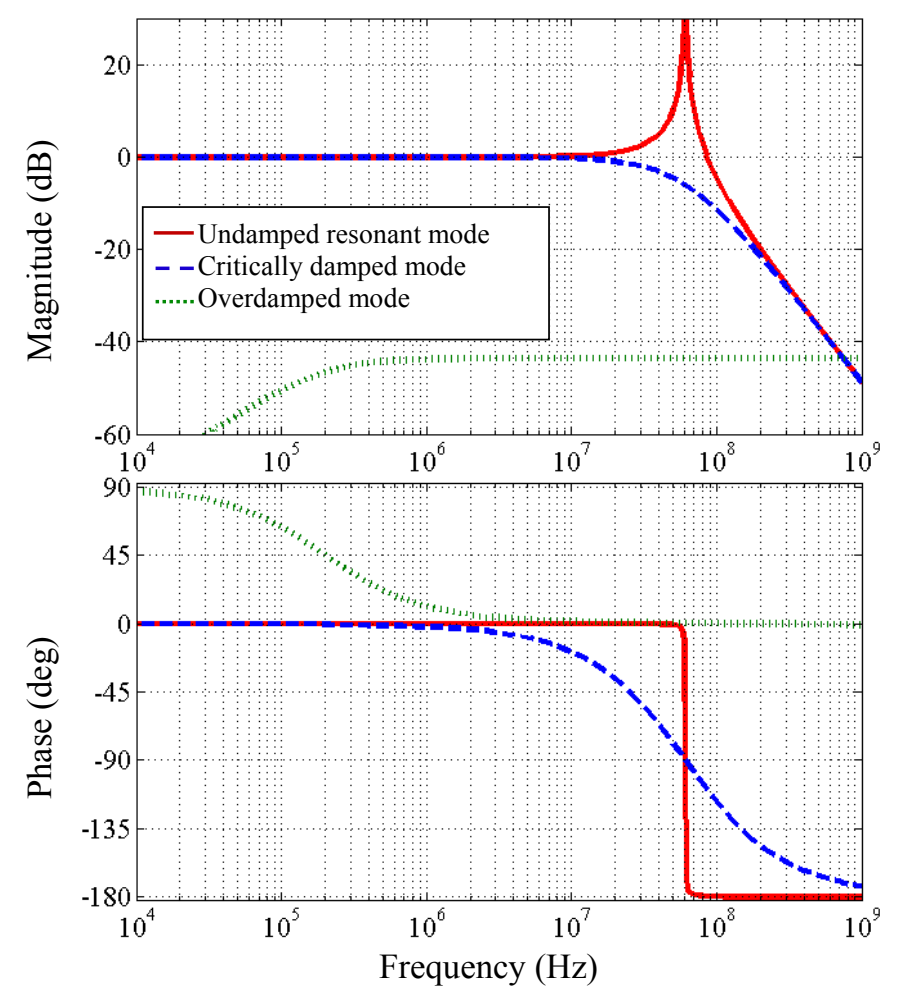

Fig. 9. Frequency plots of different Rogowski coil configurations, for the resonant and damped operating mode sensors $f_{\text {res }}=50 \mathrm{MHz}$. Plot has been normalized to represent the integrated sensor transfer rate of $1(0 \mathrm{~dB})$.

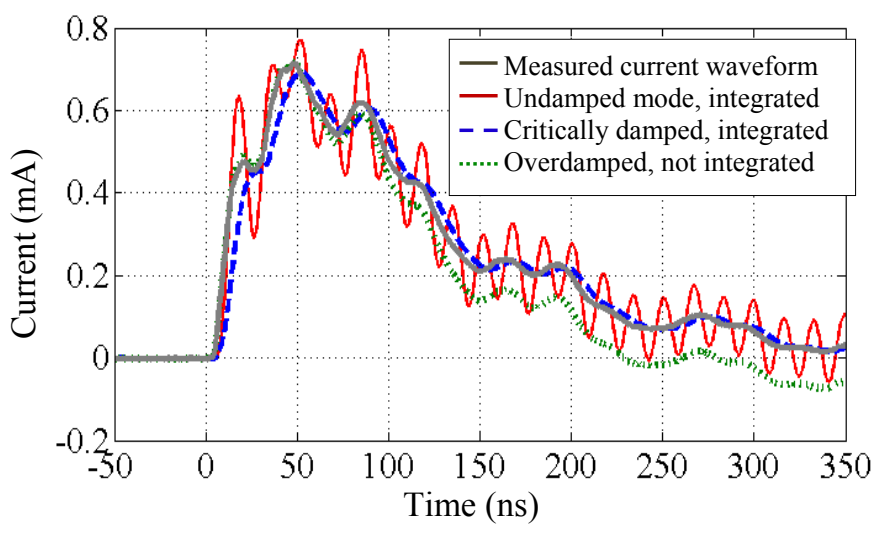

Fig. 10. Comparison of measured current waveforms using sensors with different operating modes. The sensors with resonant and damped mode of operation have resonant frequency $f_{\text {res }}=50 \mathrm{MHz}$.
Comparative frequency plots for phase and magnitude have been presented in Fig. 9, for the ideal sensor cases systems with integrated output.

A pulsed signal consists of a large number of high frequency components. This means that the sensors should have a wide operating bandwidth to capture the pulse accurately. Bandwidth in this meaning is the frequency range, where the sensor is providing relatively high sensitivity with minimum output phase shift (preferably 0). This can be achieved by integrating the output signal of the sensor (see Fig. 9). It can be seen that for frequencies higher than the resonant frequency, the magnitude transfer rate will drop sharply. The transfer rate drop of $-40 \mathrm{~dB} / \mathrm{dec}$ (100 times per frequency increase 10 times) actually presents a second-order filtering effect. Therefore, the frequency components having higher frequencies than the resonant frequency are practically filtered out from the result. In Fig. 8 it can be seen that the minimum phase shift is available for the integrated output of the sensors operating with resonant and damped output and for the overdamped, non-integrated output. This is valid until the resonant frequency $f_{\text {res }}$, above which the phase transfer angle will change by 180 degrees. Due to this, the highest frequency for the sensor to carry out measurements would be limited by the resonant frequency.

For more practical approach a comparison of measurement of the sample PD pulse (see Fig. 2) using three different modes of operation described above can be observed (see Fig. 10). This comparison of outputs of different modes is provided as a simulation in perfect conditions. It should be taken into account that in the real world applications, it is more difficult to guarantee the ideal operating conditions and therefore the accuracy will not reach the levels proposed theoretically.

From Fig. 10, it can be observed, that the resonant mode operation is not feasible in the real world application where high accuracy is required. As a result of the resonance, single frequency component is particularly emphasized, seen as clear sinusoidal oscillation. Here heavy oscillations are included even in the integrated result of the output, therefore making it difficult to observe the true waveform of the PD transient. It is more feasible to use a sensor with critically damped operating mode and integrated output. The output of such system will yield practically the same waveform as the measured current.

It can be also seen that the overdamped mode sensor provides excellent high-frequency response and matches the initial rising edge of the pulse very well. However, due to limited low-frequency response, the measurement result will have a deviation from the actual current measured in the end of the pulse.

\section{LABORATORY VERIFICATION OF OPERATION}

A prototype Rogowski coil was tested in the laboratory to verify the operating mode selection criteria. The set-up used for the experiment has been presented in Fig 11. A PD pulse was injected from a PD calibrator and the pulse was measured using two types of inductive sensors. A calibrated high frequency current transformer (HFCT) with a bandwidth of 
$80 \mathrm{MHz}$ was used for the reference measurements. The same PD pulse signal measurement was carried out with a designed prototype of Rogowski coil having standalone self-resonant frequency $f_{\text {res }}$ of $60.7 \mathrm{MHz}$ and with the measurement system connection this dropped close to $50 \mathrm{MHz}$, similar to simulations presented above. Comparison of the operating modes of the same Rogowski was done by selecting two input current pulses that were most similar to each other. This was not a difficult task, as the PD calibrator output was repeating basically identical pulses in the output. The detailed sensor and system parameters used during the tests have been provided in Table I.

As described above the sensor output has to be processed in order to restore the original waveform of the measured current. The integration operation was carried out numerically after the sensor output was acquired. The output voltage of the first stage of the measurement system, this is the sensor output captured using a digital storage oscilloscope (DSO), is presented in Fig. 12. It can be seen that the sensor operating with resonant mode did produce high portion of oscillations in the coil output. The output of a sensor operating with damped mode however provided a signal where the value differential to the measured current can be recognized.

The results of the measurement after the integration operation have been presented in Fig. 13. The results are quite similar to the theoretical simulations presented in Fig. 10. Even after the integration procedure, the sensor with resonant operating mode presents oscillations in the final measurement result. This will also make the comparison of different captured pulses rather complicated in the signal processing stages. The critically damped mode sensor final result after the integration procedure shows a very good match between the measured current pulse and output waveforms.

TABLE I.

CHARACTERISTICS OF THE ROGOWSKI COIL USED FOR THE TEST SCENARIO

\begin{tabular}{|c|c|}
\hline Parameter & Value \\
\hline Inductance $L_{S}$ & $1.2 \mu \mathrm{H}$ \\
\hline Capacitance $C_{S}$ & $5.7 \mathrm{pF}$ \\
\hline Measurement system capacitance $C_{M}$ & $2.4 \mathrm{pF}$ \\
\hline Critical damping resistance value $R_{T}$ & $190 \Omega$ \\
\hline
\end{tabular}

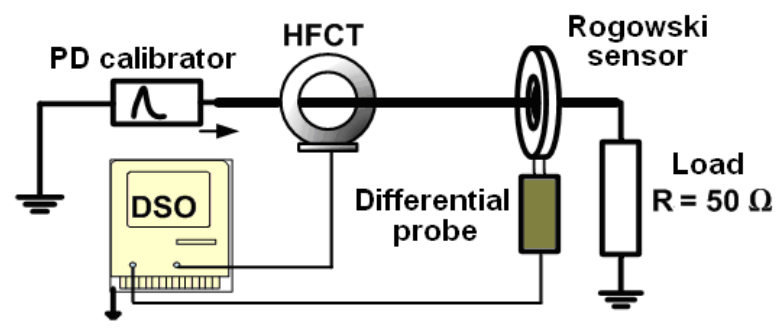

Fig. 11. Measurement set-up for verification of Rogowski sensor performance.

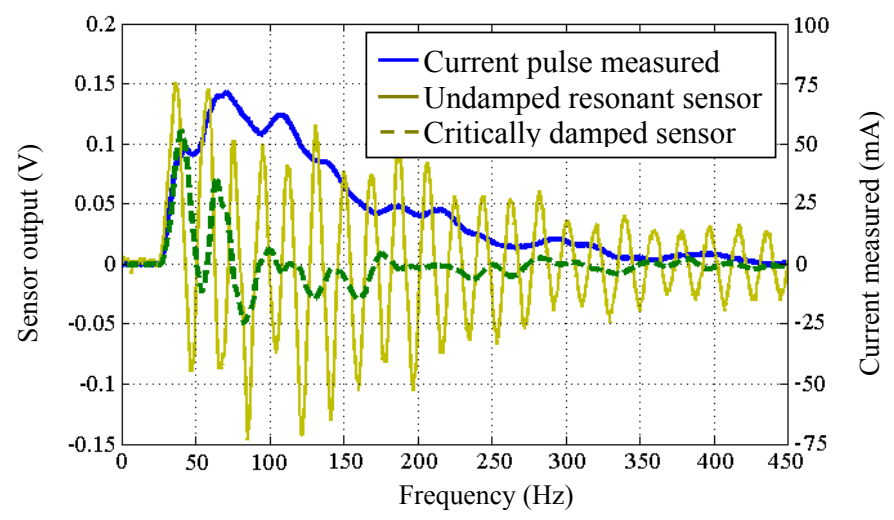

Fig. 12. Comparison of output voltages measured in the output of the inductive sensor, while measuring a current pulse using different sensor operating modes.

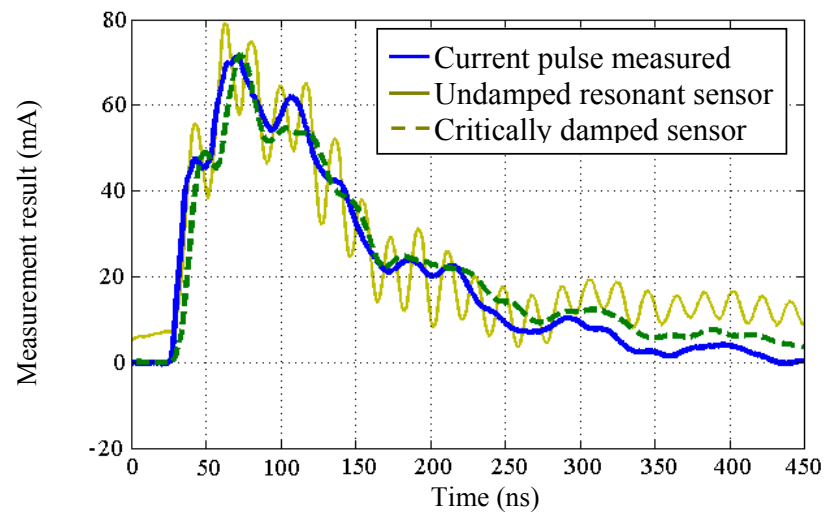

Fig. 13. Comparative final measurement results waveforms, after performing the sensor output voltage signal integration.

\section{DISCUSSION AND CONCLUSIONS}

After the signal has been acquired from the physical measurements, it can be passed on to the diagnostics system consisting of more complex data storage and processing units. Detailed analysis of the captured signal regarding phase, amplitude, pulse width and charge contained in the pulse determines the defect type, location, evolution of the activity could be detected and identified. However, for this, comparison of different pulses has to be on a very good level and the accuracy of the measurements is critical.

In the discussion above, it was presented that the sensor operating mode, dependent on the components of the measurement system, is critical for the accuracy of the measurement. The output of the sensor with resonant operating mode did differ from the reference measurement significantly, with clear emphasis of the signal component with resonant frequency. Therefore, lack of resonance of the output of the sensor has to be considered a requirement that has to be met in case accurate measurement of the PD pulses is required. In the laboratory verification of the PD measurement performance of the Rogowski air-core inductive sensor, it was witnessed that the damped mode operating mode sensor with integration of the output presented a waveform for very close to the PD pulse current waveform measured with the HFCT. 
It has to be considered, that guaranteeing the correct operating mode of the sensor is in real world applications not the only requirement, but in addition much attention should be paid to the upper and lower bandwidths of the sensor and measurement system. The bandwidth required to capture the pulse accurately is directly dependent on the pulse parameters and more detailed analysis on the measurement accuracy with limited bandwidth conditions has been presented in [17].

The on-line condition monitoring system employing the monitoring of the partial discharge activity has been rather expensive to implement and has been considered for application to monitor the most critical and expensive components like the transformers [18]. With the help of accurate and cheap sensors, the overall condition monitoring system would be also cheaper. This opens up new possibilities to implement the different condition monitoring systems more widely in the distribution networks, where it would have been too expensive to use previously. This way, the reliability of the power distribution networks could be raised to significantly higher levels.

\section{REFERENCES}

[1] A. Lvovs, A. Mutule, "Customer Dissatisfaction Index and Its Improvement Costs", Energetika un elektrotehnika Scientific Journal of RTU, Iss. 4, Vol. 27, 2010, pp. $21-26$

[2] F. Wester, E. Gulski, J. Smit, E. Groot, "Aspect of On-line and Off-line PD Diagnosis of Distribution Power Cables", 2002 IEEE International Symposium on Electrical Insulation, pp. $553-556$.

[3] S.A.Boggs, "Partial Discharge-III Cavity Induced PD in Solid Dielectrics", IEEE Electrical Insulation Magazine, Volume: 6, Issue: 6, pp.11-16, 1990.

[4] Yao Xiao, Jun Fu, Bin Hu, Xiaoping Li, Chunnian Deng, "Problems of voltage transducer in harmonic measurement", IEEE Transactions on Power Delivery, Vol. 19, No. 3, Jul 2004, pp. 1483 - 1487.

[5] G. Stone, "Importance of bandwidth in PD measurement in operating motors and generators", IEEE Transactions on Dielectrics and Electrical Insulation, Vol. 7 , Iss. 1, Feb 2000, pp. 6-11.

[6] G.M. Hashmi, M. Lehtonen, M. Nordman, "Calibration of on-line partial discharge measuring system using Rogowski coil in coveredconductor overhead distribution networks", IET Science, Measurement \& Technology, Vol. 5, Iss. 1, Jan 2011, pp. 5-13.

[7] Xin Li, Chengrong Li, Wei Wang, Bin Wei, Weijiang Wan, "Partial discharge measurement in XLPE cable joint by using VHF sensor", 2004 IEEE International Conference on Solid Dielectrics, ICSD 2004, Vol.2, pp. $669-671$

[8] G. Robles, J.M. Martinez-Tarifa, M. V. Rojas-Moreno, J. Sanz-Feito, "Inductive Sensor for Measuring High Frequency Partial Discharges Within Electrical Insulation", IEEE Transactions on Instrumentation and Measurement, Vol. 58, Iss. 11, Nov 2009, pp. 3907 - 391.

[9] Baocheng Wang, Deyu Wang, Weiyang Wu, "A Rogowski coil current transducer designed for wide bandwidth current pulse measurement", 2009 IEEE 6th International Power Electronics and Motion Control Conference, IPEMC '09, pp. 1246 - 1249.

[10] P. Valatka, V. Sučila, G. Daukšys, "Investigation of the Voltage Influence on Partial Discharge Characteristic Parameters in Solid Insulation", Electronics and Electrical Engineering, No.10 (106), 2010, pp. $63-66$.

[11] G.J. Paoletti, A. Golubev, "Partial discharge theory and technologies related to medium-voltage electrical equipment", IEEE Transactions on Industry Applications, Vol. 37, Jan/Feb 2001, pp. 90 - 103.
[12] N. Ida, "Engineering Electromagnetics" (Second Edition), Springer New York, 2004

[13] M. Rezaee, H. Heydari, Mutual inductances comparison in Rogowski coil with circular and rectangular cross-sections and its improvement; 3rd IEEE Conference on Industrial Electronics and Applications, 2008 (ICIEA 2008), pp. $1507-1511$

[14] L. Kütt, J. Järvik, H. Mõlder, J. Kilter, S. Muhammad, Magnetic Current Sensor Stray Components in High Frequency Operation and their Effects, 11th Conference on Environment and Electrical Engineering (EEEIC) 2012, $6 \mathrm{p}$.

[15] M. Shafiq, L. Kütt, M. Lehtonen, T. Nieminen, G. M. Hashmi, "Parameters Identification and Modeling of High Frequency Current Transducer for Partial Discharge Measurements", IEEE Sensors Journal, Vol. 13, Iss. 3, Mar 2013, pp. 1081 - 1091.

[16] L. Kütt, M. Shafiq, J. Järvik, M. Lehtonen, J. Kilter, "Air-core inductive current sensor for fast transients measurements in distribution networks", 2012 Electric Power Quality and Supply Reliability Conference (PQ2012), $4 \mathrm{p}$.

[17] L. Kütt, M. Shafiq, M. Lehtonen, H. Mõlder, J. Järvik, "Sensor Resonance and its Influence on the Measurement Results of Fast Transients", 2013 International Conference on Power Systems Transients (IPST2013), $7 \mathrm{p}$

[18] N. Breners, N. Skobeleva, "Mathematical Models for Choice of Measures on Functioning Reliability Increase of Power Transformers", Energetika un elektrotehnika Scientific Journal of RTU, Iss. 4, Vol. 27, 2010 , pp. $64-68$.

Lauri Kütt is received his Ph.D in 2012 from Tallinn University of Technology for the thesis of inductive sensors for measurements of fast pulsed transients. Currently he is working as a post-doc researcher in Department of Electrical Engineering in Aalto University School of Electrical Engineering, Finland. His current research topics include partial discharge measurements and power quality in distribution networks. E-mail: lauri.kutt@aalto.fi

Postal address: Department of Electrical Engineering, Aalto University School of Electrical Engineering, Otakaari 5, 01250 Espoo, Finland.

Muhammad Shafiq received his M.Sc degree in Electrical Engineering from University of Engineering and Technology (UET), Lahore, Pakistan in 2007. He is currently working as a post-graduate researcher in Department of Electrical Engineering in Aalto University School of Electrical Engineering, Finland. His field of work is related to partial discharge measurements and online insulation condition monitoring using the partial discharge measurements. E-mail: muhammad.shafiq@aalto.fi

Postal address: Department of Electrical Engineering, Aalto University School of Electrical Engineering, Otakaari 5, 01250 Espoo, Finland.

Matti Lehtonen received his D.Sc. (Tech) degree from Tampere University of Technology, Tampere, Finland, in 1992 and is working as a professor in Department of Electrical Engineering in Aalto University School of Electrical Engineering, Finland. His fields of research include distribution network reliability, power quality and planning of distribution supply. E-mail: matti.lehtonen@aalto.fi

Postal address: Department of Electrical Engineering, Aalto University School of Electrical Engineering, Otakaari 5, 01250 Espoo, Finland.

Heigo Mõlder received his Ph.D degree from Tallinn University of Technology for the thesis in topics of electromagnetic modeling of complex systems. Currently he is working as a researcher in Department of Electrical Engineering in Tallinn University of Technology, Estonia. He is working with topics of electromagnetic compatibility and electromagnetic field modeling. E-mail: heigo.molder@ttu.ee

Postal address: Department of Electrical Engineering, Tallinn University of Technology, Ehitajate tee 5, 19086 Tallinn, Estonia.

Jaan Järvik received his doctoral candidate degree in 1971 from Leningrad Polytechnic Institute (now St.Petersburg Technical University). Currently he is working as a professor in Department of Electrical Engineering in Tallinn University of Technology, Estonia. He has long experience in working with topics of reactive power compensation, power supply reliability and fundamentals of electrical engineering. E-mail: jaan.jarvik@ttu.ee

Postal address: Department of Electrical Engineering, Tallinn University of Technology, Ehitajate tee 5, 19086 Tallinn, Estonia. 